\title{
RECURRENT PRIMARY MESENTERIC LIPOSARCOMA
}

\author{
Srijan Malla ${ }^{1}$, Jyoti Rayamajhi ${ }^{1}$, Sushil Bahadur Rawal ${ }^{1}$, Naresh Kumar Giri ${ }^{1}$ \\ ${ }^{1}$ Department of Surgery, Shree Birendra Hospital
}

\begin{abstract}
Liposarcoma occurring in the mesentery primarily is a rare entity, scarcely reported in world literature. Here, we report one such case that recurred thrice in spite of complete resection and adjuvant therapy. A literature review on the management of such an aggressive tumor is also outlined.
\end{abstract}

Key words: mesenteric liposarcoma, soft tissue Sarcoma.

\section{INTRODUCTION}

Liposarcoma is one of the most common soft tissue sarcoma occuring in limbs, retroperitoneum and abdominal cavity of adults. However primary mesenteric liposarcomas are very rare neoplasms. The first case in the English literature was described by Manson in $1951 .{ }^{1}$ Only 24 cases of this neoplasm are described in literature. Here we report a case of Recurrent Primary Mesenteric Liposarcoma we encountered in our hospital.

\section{CASE REPORT}

A 44yr old gentleman, presented at the Surgical out patient department with complaints of a painless, gradually increasing abdominal lump for 2 months. He gave no history of altered bowel habits or urinary symptoms. Examination revealed a freely mobile, non tender, firm intra-abdominal mass measuring $16 \times 12 \mathrm{~cm}$ mainly located in the right lumbar and umbilical region. Lab investigations revealed no abnormalities.

Ultrasonography of the abdomen showed a mass lesion on the right side of abdomen consistent with a malignant mass lesion- likely to be a soft tissue sarcoma. Further investigation with a CECT abdomen indicated a heterogenous intraabdominal soft tissue mass lesion suggestive of a teratodermoid with a diiferential diagnosis of a Gastrointestinal Stromal Tumor (Figure 1). A USG guided FNAC remained negative for malignant cells revealing bands of mesothelial cell clusters and smooth muscle fragments.

With the probable diagnosis of a mesenteric soft tissue tumor, laparotomy was performed and a large mass in mesentery of ileum $20 \times 15 \times 10 \mathrm{~cm}$ with vascular supply from mesenteric vessels resected (Figure 2). Approx 1 foot bowel excised was sacrificed due to doubtful viability following resection of the mesenteric mass.

Histopathology revealed undifferentiated Liposarcoma, histological grade II with tumor free margins and no vascular or lymphatic invasion. Pathologically it was staged as $\mathrm{T} 2 \mathrm{~b} \mathrm{Nx} \mathrm{Mx}$.

After discharge the patient was on 3 monthly follow up with USG. However after approximately 1 year, the patient was again readmitted with similar complaints and was found to have a recurrence of the mass on USG. Relaparotomy showed multiple mesenteric mass in distal ileum [15X $15 \mathrm{~cm}]$, proximal jejunum [ $6 \times 8 \mathrm{~cm}]$, transverse mesocolon $[6 \times 6]$. All these masses were resected with resection anastamosis of part of the jejunum. The patient was subjected to 6 cycles of adjuvant chemotherapy and kept on 3 monthly follow up regime. However, the patient was re-admitted a third time with similar complaints after another 1 year. Laparotomy this time revealed multiple masses in the transverse mesocolon in addition

\author{
Correspondence: \\ Dr. Srijan Mall \\ Department of Surgery, Shree Birendra Hospital \\ E-mail: srijan@gmail.com \\ Cell No.: 9851064964
}


to the mesentry of jejunum. A successful resection of the tumors, sacrificing part of jejunum to obtain adequate tumor free margins was done. Adjuvant chemotherapy was once again instituted.

To our dismay, the patient came back after exactly one year with recurrence. Laparotomy revealed multifocal tumor with matted small and large bowel (Figure 3). It was deemed unresectable without sacrificing the entire bowel. Hence, surgery was abandoned and the patient referred to oncologist for chemotherapy.

\section{DISCUSSION}

Liposarcoma is the second most common soft tissue sarcoma following malignant fibrous histiocytoma, accounting for approximately $16-18 \%$ of all soft tissue tumors, and frequently occurs in the lower extremities and the retroperi-toneum. ${ }^{2}$ Primary tumors of mesentery are uncommon. To our knowledge, only 24 cases of liposarcoma occurring in the mesentery have been published in world literature. ${ }^{3}$ It is histologically defined as a tumor composed of lipoblasts.

WHO has classified this disease into 5 Groups - well differentiated, myxoid, round, pleomorphic and undifferentiated. ${ }^{4}$

The mode of clinical presentation is dependent on the size and exact location of the tumour. The physical finding of a mass that is easily movable in all directions is frequent and has some diagnostic utility

Computed tomography and MRI are helpful for diagnosis and for planning therapy. ${ }^{5}$ Each histologic subtype of abdominal liposarcoma show different CT attenuation or MR imaging signal intensity characteristics. ${ }^{6}$

Complete surgical resection at the time of primary presentation is likely to give a chance for long-term survival as well as distant recurrence-free survival. ${ }^{7}$ The treatment of choice for such liposarcoma is surgical resection with sufficient surgical margin, often followed by radiation and/or adjuvant chemotherapy for tumors with a high risk of relapse, such as for large tumors or lowgrade tumors.
In our case, the patient kept developing recurrences in spite of resections with wide surgical margins and adjuvant chemotherapy. What is also striking is these recurrences developed almost exactly one year after the previous surgery despite being on 3 monthly follow up with USG abdomen to detect recurrences. The sites of recurrences were also varied- the initial location was confined to ileal mesentry whereas in subsequent episodes these appeared in jejunal mesentry as well as in transverse mesocolon. Such aggressive multifocal tumor, recurring so rapidly even after aggressive surgical resection and chemotherapy has not been described. Unfortunately, the patient ultimately succumbed to the disease after it was deemed unresectable after the fourth recurrence leaving us to wonder- was there anything else that could have been done?

\section{REFERENCES}

1. Manson JMS. Mesenteric liposarcoma. A rare case of intestinal obstruction. Br J Surg 1951; 38: 394-396.

2. Kransdorf MJ. Malignant soft tissue tumors in a large referral population: distribution of diagnosis by age, sex, and location. Am J Roentgenol 194: 129-134, 1995.

3. Hirakoba $\mathrm{M}$ et al. Primary mesenteric liposarcoma successfully diagnosed by preoperative imaging studies. Intern Med. 2007; 46(7):373-5.

4. Christopher DM. WHO classification of tumors. Pathology and genetics: tumors of soft tissue and bone. Lyon, France: IARC Press, p.35-46, 2002.

5. Munk PL, Lee MJ, Poon PY, Goddard KJ, Knowling MA, Hassell PR. Computed tomography of retroperitoneal and mesenteric sarcomas: a pictorial essay. Can Assoc Radiol J 1996; 47: 335-341.

6. Kim T, Murakami T, Oi H, Tsuda K, Matsuhita M, Tomoda K, et al. CT and MR imaging of abdominal liposarcoma. AJR Am J Roentgenol 1996; 166: 829-833.

7. Stojadinovic A, Leung DH, Hoos A, Jaques DP, Lewis JJ, Brennan MF. Analysis of the prognostic significance of microscopic margins in 2,084 localized primary adult soft tissue sarcomas. Ann Surg 2002;235:424-34. 


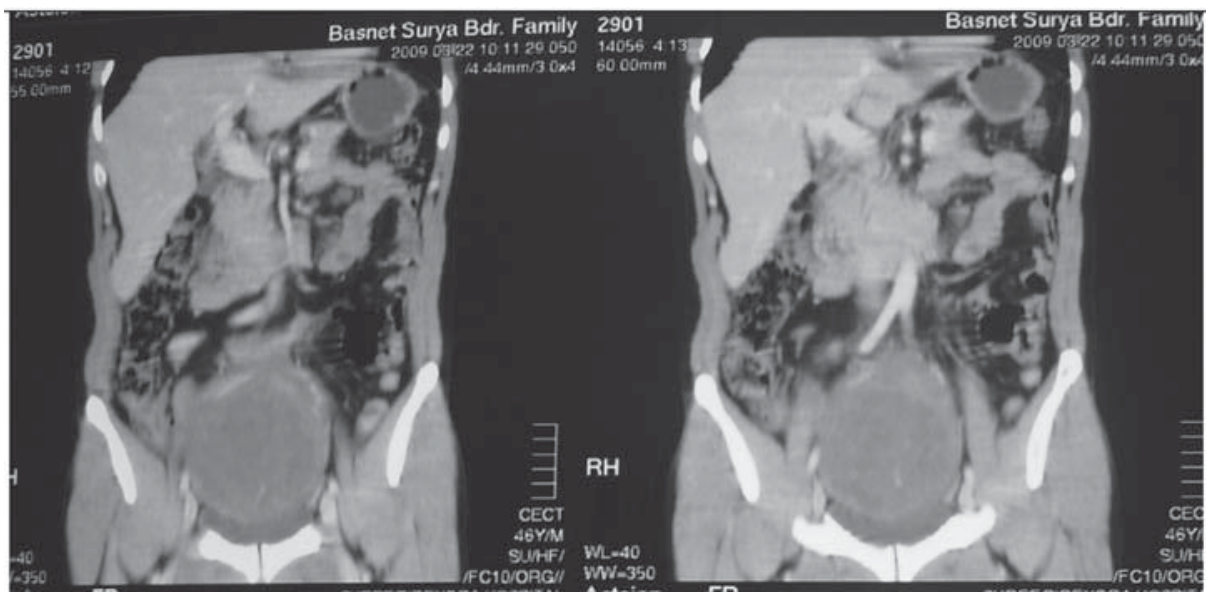

Figure 1. CECT abdomen indicated a heterogenous intraabdominal soft tissue mass.
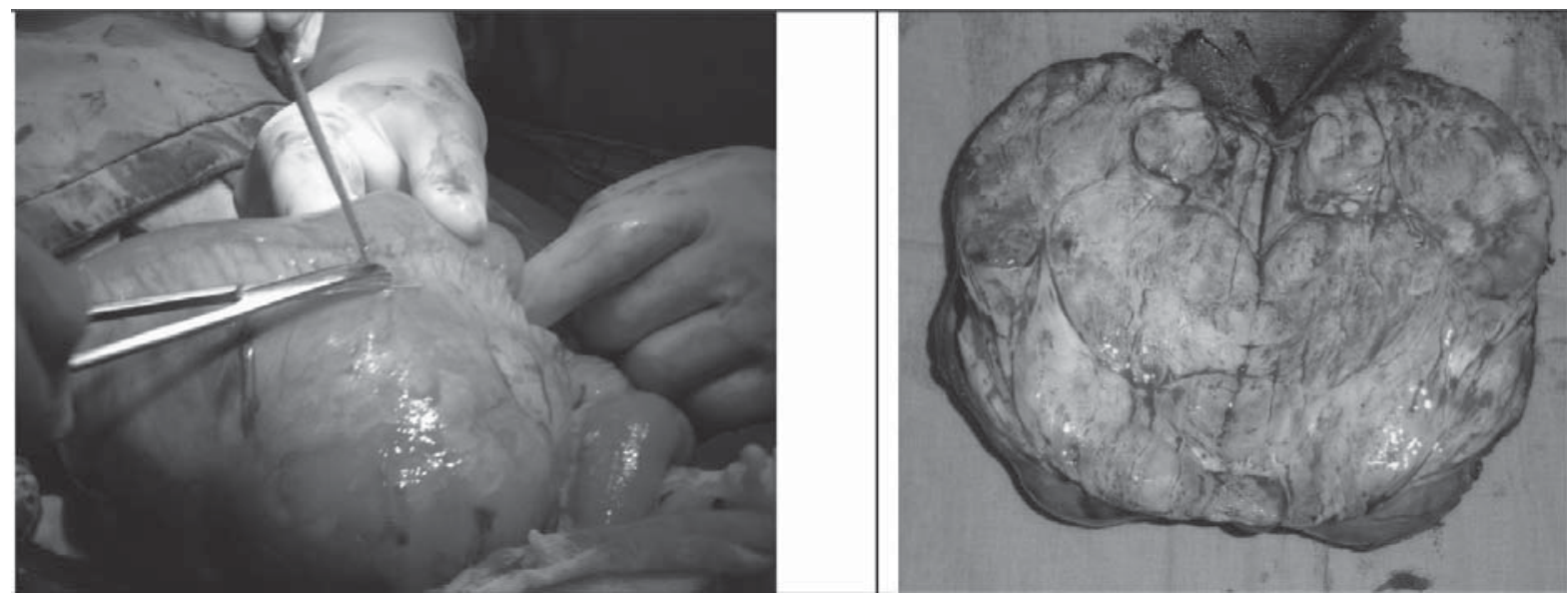

Figure 2. A. large mass in mesentery of ileum $20 \times 15 \times 10 \mathrm{~cm}$ with vascular supply from mesenteric vessels. B. Resected mass in section
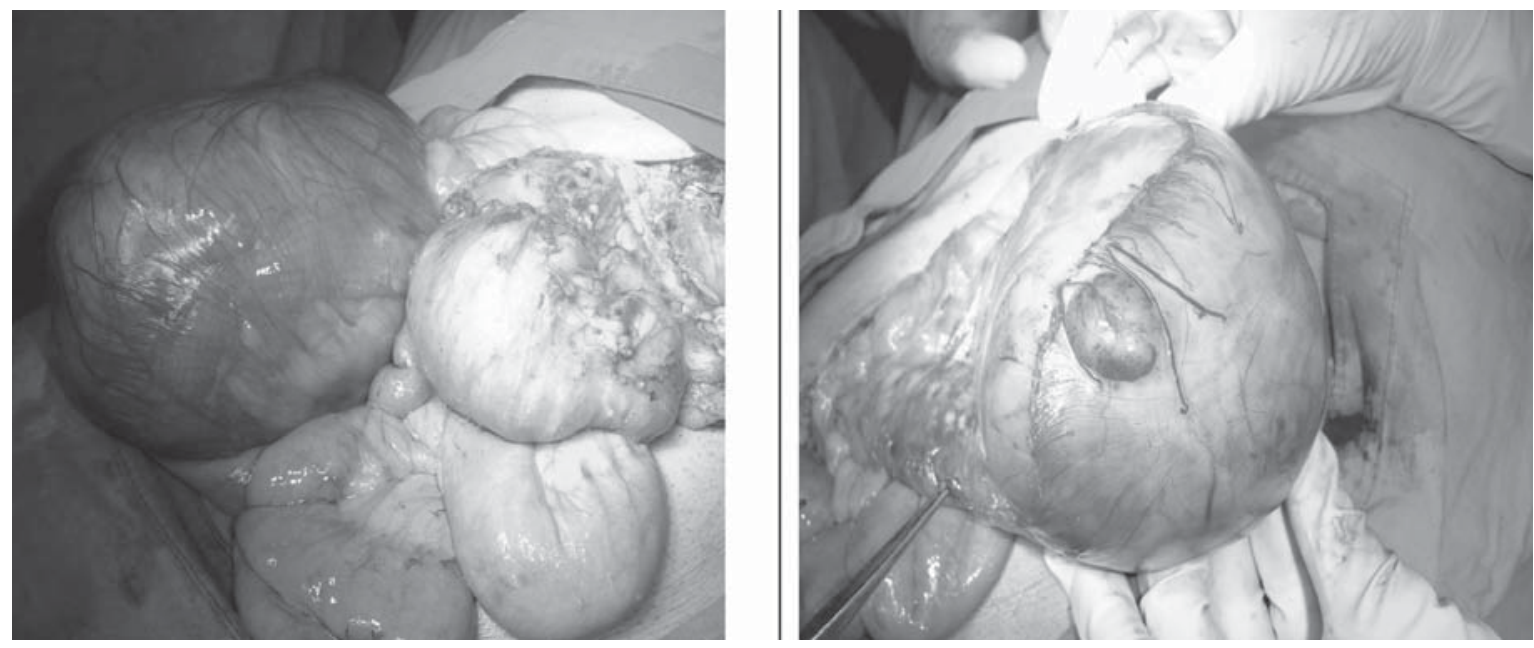

Figure 3. Laparotomy revealed multifocal tumor with matted small and large bowel. 